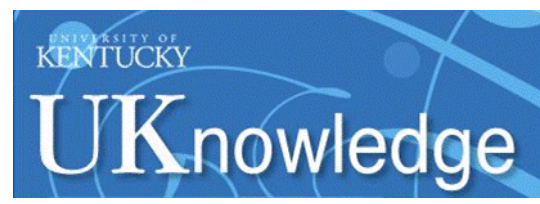

University of Kentucky

UKnowledge

6-1-1992

\title{
Water Masers in W49N - The Youngest Stellar Jet?
}

Mordecai-Mark Mac Low

NASA Ames Research Center

Moshe Elitzur

University of Kentucky, moshe@pa.uky.edu

Follow this and additional works at: https://uknowledge.uky.edu/physastron_facpub

Part of the Astrophysics and Astronomy Commons, and the Physics Commons

Right click to open a feedback form in a new tab to let us know how this document benefits you.

\section{Repository Citation}

Low, Mordecai-Mark Mac and Elitzur, Moshe, "Water Masers in W49N - The Youngest Stellar Jet?" (1992). Physics and Astronomy Faculty Publications. 230.

https://uknowledge.uky.edu/physastron_facpub/230

This Article is brought to you for free and open access by the Physics and Astronomy at UKnowledge. It has been accepted for inclusion in Physics and Astronomy Faculty Publications by an authorized administrator of UKnowledge. For more information, please contact UKnowledge@lsv.uky.edu. 


\section{Water Masers in W49N - The Youngest Stellar Jet?}

\section{Digital Object Identifier (DOI)}

http://dx.doi.org/10.1086/186444

\section{Notes/Citation Information}

Published in The Astrophysical Journal Letters, v. 393, no. 1, p. L33-L36.

(C)1992. The American Astronomical Society. All rights reserved.

The copyright holder has granted permission for posting the article here. 
ThE ASTROPHYSICAL JoURNAL, 393:L33-L36, 1992 July 1

(C) 1992. The American Astronomical Society. All rights reserved. Printed in U.S.A

\title{
WATER MASERS IN W49N-THE YOUNGEST STELLAR JET?
}

\author{
Mordecai-Mark MaC Low ${ }^{1}$ AND Moshe Elitzur 2,3 \\ Space Science Division, NASA Ames Research Center, MS 245-3, Moffett Field, CA 94035-1000 \\ Received 1992 January 17; accepted 1992 April 16
}

\begin{abstract}
Observations by Gwinn, Moran, and Reid of the proper motions of water masers in W49N show that they have an elongated distribution expanding from a common center. Features with high space velocity only occur far from the center, while low-velocity features occur at all distances. We propose that water masers in star-forming regions occur in expanding shells swept up by high-velocity winds from young, massive stars during the early phases of the expansion. In W49N, confinement of the bubble by a density distribution with an axial cavity can explain both the velocity field and the shape of the maser distribution. We present a fully dynamical calculation of the expanding bubble which provides a satisfactory fit for the observations and suggests that this system is only $\sim 250 \mathrm{yr}$ old. Thus these observations may show the very first stages of the formation of a jet from a young stellar object.
\end{abstract}

Subject headings: circumstellar matter - masers - stars: formation

\section{INTRODUCTION}

Newly formed stars usually have powerful outflows traced by a number of species. One of the tracers of such outflows is strong $\mathrm{H}_{2} \mathrm{O}$ maser emission, widely regarded as a signpost of star formation. There appears to be general agreement that $\mathrm{H}_{2} \mathrm{O}$ masers, high-velocity emission in various atomic and molecular lines (including $\mathrm{CO}$, vibrational $\mathrm{H}_{2}$, etc.), optical jets, and Herbig-Haro objects are different manifestations of the same phenomenon: high-velocity mass outflow in the last stages of pre-main-sequence evolution of newly formed stars (Lada 1985; Genzel 1986). However, it is not yet known how and when star formation triggers $\mathrm{H}_{2} \mathrm{O}$ maser action. A telling example is provided by $\mathrm{W} 49$, which contains the most luminous $\mathrm{H}_{2} \mathrm{O}$ maser source in our Galaxy. High-resolution observations by Welch et al. (1987) show that the core of this star-forming region contains a $2 \mathrm{pc}$ ring of at least 10 distinct ultracompact $\mathrm{H}$ II regions, each containing at least one $\mathrm{O}$ star. The $\mathrm{H}_{2} \mathrm{O}$ maser emission occurs in just one of these $\mathrm{H}$ II regions. At least two molecular outflows much larger than the $\mathrm{H}_{2} \mathrm{O}$ maser source and with different orientations also lie in the region.

A recent study by Gwinn, Moran, \& Reid (1992, hereafter GMR) provides a detailed picture of the maser field in W49N. From VLBI observations at five epochs, GMR find that the masers lie in an elongated, cylindrically symmetric distribution, with the highest space velocity masers at the ends. They obtain a reasonable fit to the overall velocity field for a model that assumes pure expansion from a central object, though the residuals exceed the turbulent velocity of $10 \mathrm{~km} \mathrm{~s}^{-1}$. The inclusion of an effective rotation produces the very low-dispersion fit shown in our Figure 1. Although the physical reality of the rotation remains unclear, the coherent curve found by including it argues for some form of shear or rotational flow. Leaving it out simply increases the scatter of the points in Figure 1 without removing the sudden increase of velocity at high radius. GMR interpret the maser distribution as a biconical

\footnotetext{
1 Also Department of Astronomy, University of California at Berkeley.

${ }^{2}$ Also Department of Physics and Astronomy, University of Kentucky at Lexington.

${ }^{3}$ National Research Council Senior Research Associate.
}

expansion from a single common center, with acceleration to velocities of $\gtrsim 200 \mathrm{~km} \mathrm{~s}^{-1}$ at the outer edges of the maser cluster. In this interpretation, single maser features correspond to blobs of material that move at low velocity toward the edge of the region, a journey that takes $\sim 10^{3} \mathrm{yr}$, where they experience strong acceleration. GMR mention nozzle mechanisms, special arrangements of shock waves, and radiative acceleration as possible candidates for the cause of the relatively sudden acceleration at $\sim 10^{17} \mathrm{~cm}$.

\section{CONCEPT}

We propose a different interpretation for the observed velocity field. As noted above, young stars usually have a strong outflow associated with them. Such an outflow must be driven by a strong stellar wind that will sweep the surrounding gas into a shell. The structure of these objects, called stellars wind bubbles, has been studied in detail in the diffuse interstellar medium (e.g., Castor, McCray, \& Weaver 1975; Weaver et al. 1977; Steigman, Strittmatter, \& Williams 1975) and is shown in Figure 2. The free-flowing wind stops in a strong shock, producing a region of shocked wind gas. This shocked gas drives another shock into the ambient gas, sweeping up material into a thin, radiatively cooled shell. In the high densities of a star-forming core, a shell will form within a few years after the wind begins. Figure 2 shows the two shocks as thicker lines. In our interpretation, the $\mathrm{H}_{2} \mathrm{O}$ masers are produced behind the outer radiative shock, as described, for example, by Elitzur, Hollenbach, \& McKee (1989, hereafter EHM). Individual spots correspond to velocity coherent regions in the shell. The velocity field of the maser features traces the velocity profile of the expanding shell surface. The small spread in expansion velocities at any given radius displayed by the data presented in Figure 1 provides support for this interpretation.

As a simple geometric illustration of a shell velocity field that can actually produce the observed relation of radius to velocity, consider a cylindrical shell whose walls expand slowly while its ends expand quickly. The surface of the shell has low velocities at all radii from the center and high velocities only at large radii, similar to what is observed in W49N. In such a configuration, single maser spots need not travel and accelerate individually to reach high velocities. Instead, they are part 


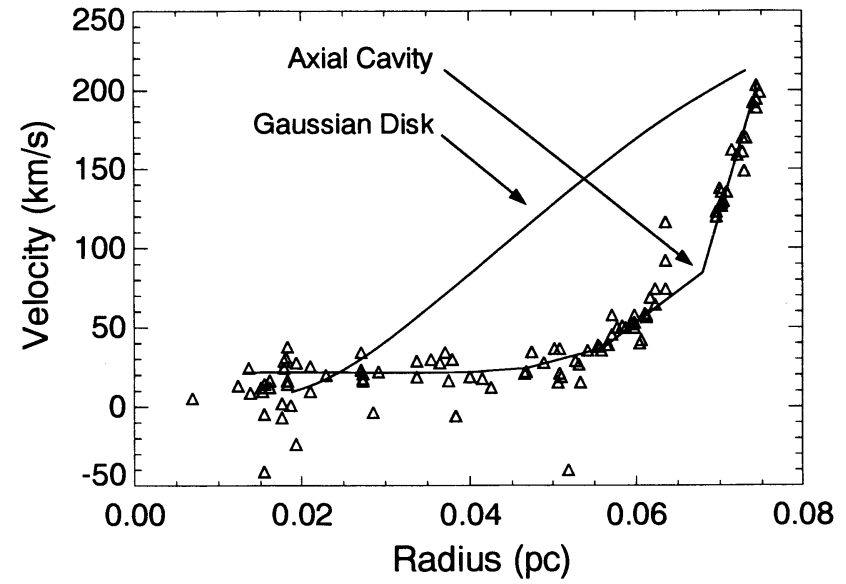

FIG. 1.-Space velocities and distances from the center of expansion of $\mathrm{H}_{2} \mathrm{O}$ masers in W49N. Triangles are data points from Fig. 5 of GMR. Solid lines show the results of full dynamical calculations of wind-driven expanding shells. The Axial Cavity line corresponds to the model described by eqn. (4) and subsequent discussion. The Gaussian Disk model has $n(z)=$ $n_{0} \exp \left[-(z / H)^{2}\right]$ with $n_{0}=10^{9} \mathrm{~cm}^{-3}$ and $H=2.8 \times 10^{16} \mathrm{~cm}$, and a wind characterized by $\dot{M}_{-3}=3.0$ and $v_{w, 8}=1.6$. The plotted curve corresponds to $800 \mathrm{yr}$ after wind onset.

of a decelerating shell, different parts of which decelerate at different rates due to the external density distribution.

Our proposal implies that the shell cannot be spherical because such a geometry would give a single radius and a single expansion velocity for all the maser features, contrary to observations. Furthermore, self-similar flows, with the location of the shell separable into $R(r, t)=f(r) R_{s}(t)$, give a linear relation of velocity to radius. This is because the velocity of the shell surface is given by $V(r, t)=f(r) V_{s}(t)$, where $V_{s}=\dot{R}_{s}$, so $V(r, t)=\left(V_{s} / R_{s}\right) R(r, t)$. Self-similar flows include inertial flows where no significant forces act on the shell. Nearly inertial flows, with only small pressure forces acting on the shell, give nearly linear relations of velocity to radius. As evident from Figure 1, linear relations contradict the observations.

Although the observed velocity field implies large deviations from spherical symmetry, bubble expansion into an isotropic medium provides useful estimates for the scale sizes of relevant quantities. Simple analytic solutions are then available in two limits, determined by the relation between the cooling time for the shocked wind gas, $t_{\text {cool }}$, and the dynamical time of the bubble, $t$. When $t_{\text {cool }}>t$, the shocked wind gas remains hot, forming a pressure-driven shell, described by Castor et al. (1975). The shell radius in a homogeneous medium is

$$
R_{s}=8 \times 10^{16} \mathrm{~cm}\left(\dot{M}_{-3} v_{w, 8}^{2} / n_{6}\right)^{1 / 5} t_{2}^{3 / 5},
$$

where $v_{w}=v_{w, 8} \times 10^{8} \mathrm{~cm} \mathrm{~s}^{-1}$ is the wind velocity, $\dot{M}=\dot{M}_{-3}$ $\times 10^{-3} M_{\odot} \mathrm{yr}^{-1}$ is its mass loss rate, $n=n_{6} \times 10^{6} \mathrm{~cm}^{-3}$ is the density of the ambient medium, and $t=t_{2} \times 100 \mathrm{yr}$ is the time after the start of the expansion. Similarly, the expansion velocity of the shell is

$$
V_{s}=160 \mathrm{~km} \mathrm{~s}^{-1}\left(\dot{M}_{-3} v_{w, 8} / n_{6}\right)^{1 / 5} t_{2}^{-2 / 5} .
$$

These scales compare favorably with the values observed in W49N when all the dimensionless parameters are of order unity. Similar scales are obtained when $t_{\text {cool }}<t$, a situation corresponding to momentum-driven shells, described by Steigman et al. (1975; see also Königl 1982).

In the diffuse interstellar medium, stellar wind bubbles go through a long pressure-driven phase before conductive evaporation (Cowie \& McKee 1977) of mass from the cold shell to the hot interior raises the interior density sufficiently that it can cool radiatively and become momentum-driven (Weaver et al. 1977). The high densities and small length scales of our problem shorten both the cooling and dynamic time scales. In a uniform velocity wind, the density at the radius of the termination shock $R_{t}$ (the inner shock in Fig. 2) varies as

$$
n\left(R_{t}\right)=9.5 \times 10^{5} \mathrm{~cm}^{-3} \dot{M}_{-3} v_{w, 8}^{-1} R_{16}^{-2},
$$

where $R_{16}=R_{t} / 10^{16} \mathrm{~cm}$, and the density of the gas after passing through that shock is a factor of 4 higher. Assuming $10 \% \mathrm{He}$ and full ionization, the temperature of this shocked gas is $T=2.9 \times 10^{7} \mathrm{~K} v_{w, 8}^{2}$. The cooling time behind the termination shock can then be evaluated from the cooling curve of Mac Low \& McCray $(1988), \Lambda(T)=10^{-22}\left[T /\left(10^{6} \mathrm{~K}\right)\right]^{-0.7}$ ergs $\mathrm{cm}^{3} \mathrm{~s}^{-1}$, yielding

$$
t_{\text {term }}=14 \text { yr } v_{w, 8}^{4.4} \dot{M}_{-3}^{-1} R_{16}^{2} .
$$

Thus the bubble actually starts out momentum driven, but it remains so only as long as $t_{\text {term }}<t \sim 3 \mathrm{yr} R_{16} / v_{w, 8}$.

\section{COMPUTATIONS AND RESULTS}

We now want to construct a physical model of a bubble that can reproduce the kinematics observed by GMR. To model the

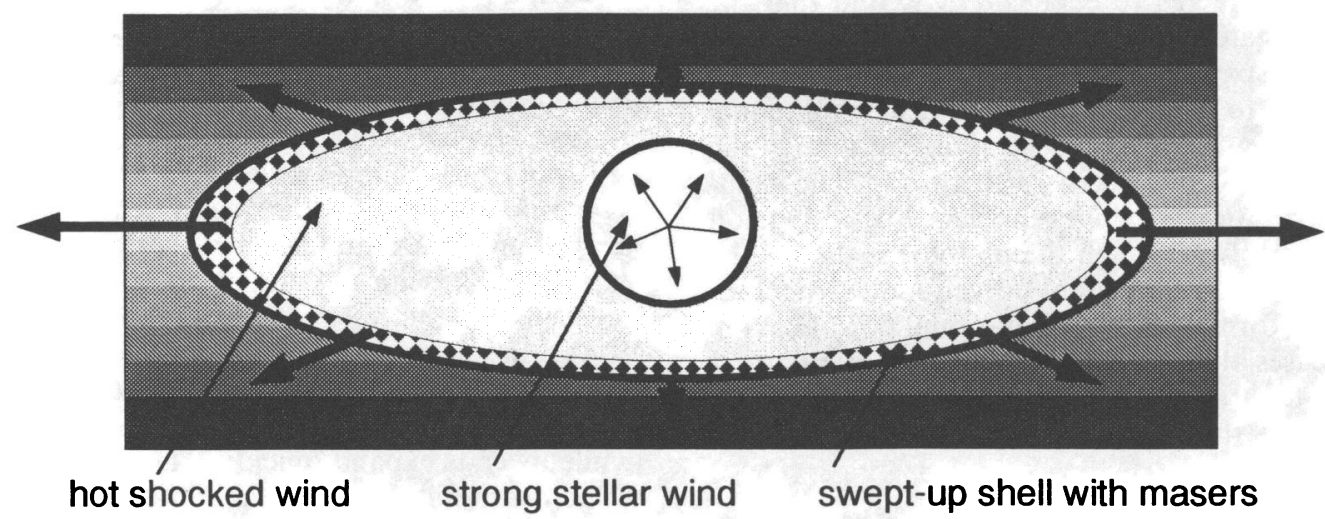

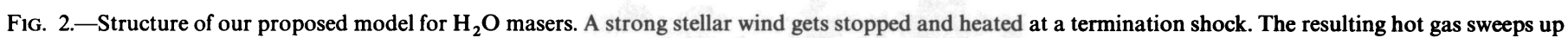

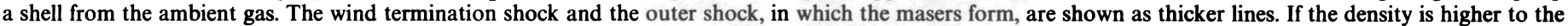
sides than toward the poles, the poles will decelerate more slowly, producing higher velocities at larger radii, as observed in W49N. 
bubble we make three assumptions. First, we assume cylindrical symmetry around the long axis, as suggested by the observations. Second, we assume that the shell of swept up ambient gas has a thickness much less than its radius. This assumption holds because the high density of the ambient gas implies that it will radiatively cool in $2 n_{6}^{0.71} \dot{M}_{-3}^{0.29} v_{w, 8}^{0.58} \mathrm{yr}$ after being shocked. Third, we assume that the interior remains isobaric, since the dynamical time exceeds the sound crossing time in the hot interior. These assumptions allow us to use a slightly modified version of the thin shell code described by Mac Low $\&$ McCray (1988). We explicitly include the radiative cooling of the interior as described in that work.

The two simplest causes of wind-driven shell elongation are a collimated wind or a nonuniform ambient density distribution. A wind collimated closer to the star than the observed scale would have to actually hit the shell in order to influence its shape, implying a momentum-driven shell. In a uniform medium, each angular segment of the shell then moves radially under the influence of a collimated radial wind of varying strength. However, the motion of each segment separately has the same time dependence, so the relation of velocity to radius remains linear, as we discussed in $\S 2$. We confirmed this result using the momentum-driven code described by Mac Low et al. (1991), eliminating collimated winds as an explanation. Masson \& Chernin (1992) also show that a collimated wind in a homogeneous medium produces a similarity solution.

From the discussion of $\S 2$, the nonlinear relation of velocity to radius excludes self-similar flows, as well as spherically symmetric density distributions. The relation also excludes any configuration in which the bulk of the shell effectively breaks from a local confinement and then coasts in an inertial flow. Most planar density stratifications admit such solutions (Kompane'ets 1960; Königl 1982), and our numerical work again confirms that such models give a nearly linear dependence of velocity on radius. For example, Figure 1 presents the plot of velocity to radius when the bubble expands in a medium with a planar Gaussian stratification along the symmetry axis, such as might be expected from a simple model of a remnant disk around the central star. While matching the high- and low-velocity ends, the displayed curve fails badly in the intermediate region. This is typical of stratified distributions, which fail to explain the observations.

Having excluded spherical and planar density stratifications, as well as collimated winds, we were led to the next simplest density distribution: a cylindrical stratification. Such a density distribution can collimate the flow, confining expansion to the axis. In order for the shell to expand most quickly along the axis, the density must increase perpendicularly. In other words, a cylindrical cavity must exist along the axis of symmetry, such as might be produced by a thick disk. We describe such cavities with a density distribution of the form

$$
n(\rho, z)=\min \left\{n_{0}\left[1+\exp \left(\frac{\rho-\rho_{0}}{H}\right)\right], n_{m}\right\},
$$

where $\rho$ is the cylindrical radius, $\rho_{0}$ and $H$ are free parameters that determine, respectively, the radius of the cavity and the steepness of its walls, and $n_{0}$ and $n_{m}$ are, respectively, the density along the cavity axis and far away from it (at $\rho-\rho_{0} \gg$ $H$ ). Using this density law, we sought solutions that fit the observations. We did not perform a full search of parameter space, but simply took the scales used in equations (1) and (2) as a starting point and changed them looking for plausible fits.
In Figure 1 we show such a model with $\dot{M}_{-3}=3$ and $v_{w, 8}=$ 1.4. The cavity is characterized by $H=10^{15} \mathrm{~cm}$ and $\rho_{0}=1.5$ $\times 10^{16} \mathrm{~cm}$, and we selected $n_{0}=2 \times 10^{6} \mathrm{~cm}^{-3}$ and $n_{m}=2$ $\times 10^{8} \mathrm{~cm}^{-3}$ to fit the low and high velocities observed. (These values roughly bound the likely range of preshock densities that give rise to bright masers in the EHM scheme.) The temperature of the ambient medium was set equal to $30 \mathrm{~K}$. We have verified that, as expected, its actual value does not affect the results since the ram pressure of the wind greatly exceeds the ambient thermal pressure. The plotted curve, which reproduces the observations rather well, corresponds to $t=250 \mathrm{yr}$ after the wind onset. The shell containing the masers has an elongated, cylindrically symmetric shape with high velocities at the ends and low velocities on the sides, as seen by GMR.

The nature of the cylindrical cavity determines the relation of velocity to distance: constant velocity at small radii with an exponential rise in velocity at large radii. The part of the shell near the equator that is moving into the dense gas with $n=n_{m}$ produces the constant velocity region. At large radii, the shell encounters lower densities (see Fig. 2), producing the exponential increase in velocity with radius. To get the observed steepness of the increase, the radius of the cavity $\rho_{0}$ had to be within $25 \%$ of the given value. Increasing either $n_{m}$ or $H$ moves the curve down and to the right. The observed peak velocity strongly constrains the mechanical luminosity of the wind $L=$ $\left(\frac{1}{2}\right) \dot{M} v^{2}$, although varying the axial density $n_{0}$ changes the needed wind values slightly.

\section{DISCUSSION}

The suggestion that $\mathrm{H}_{2} \mathrm{O}$ masers are located in expanding shells around newly formed stars has already been made in a number of observational studies (Genzel et al. 1978; Walker et al. 1978; Felli, Palagi, \& Tofani 1992). Our proposal provides a physical model for these shells without invoking any new concepts or mechanisms. It simply applies the standard bubble model of the action of a strong wind on diffuse gas to the extreme conditions near a newly formed, massive star. The outer shock of a fast-moving bubble provides an environment for the formation of $\mathrm{H}_{2} \mathrm{O}$ masers, as described, for example, in EHM, although the explanation of low-velocity $(\$ 20-30 \mathrm{~km}$ $\mathrm{s}^{-1}$ ) masers may require $\mathrm{C}$-shock calculations that are not yet available. The result is that the masers act as a powerful probe of the density distribution in the region between $10^{16}$ and $10^{17}$ $\mathrm{cm}$ from the star.

The mass-loss rate and wind velocity required by our model agree with values inferred from high-resolution mapping of molecular outflows around young stellar objects by Scoville et al. (1986). They deduce that the outflow in NGC 7538 is powered by a wind with velocity of $\sim 1000 \mathrm{~km} \mathrm{~s}^{-1}$ and massloss rate of $10^{-3} M_{\odot} \mathrm{yr}^{-1}$, comparable to the values we require. They also find that $\mathrm{W} 49 \mathrm{~N}$ has a molecular outflow, centered on a different $\mathrm{H}$ II region from the one at the center of the $\mathrm{H}_{2} \mathrm{O}$ maser activity, with an overall momentum of $3500 \mathrm{M}_{\odot} \mathrm{km} \mathrm{s}^{-1}$. A wind of the strength we infer could deposit that amount in $\sim 1000 \mathrm{yr}$, which is the expected time scale, since such an outflow only becomes observable at later stages than those we discuss. It is encouraging that another $\mathrm{H}$ II region in the ring of $\mathrm{W} 49 \mathrm{~N}$ also provides evidence for a wind of this strength. Genzel \& Downes (1977) actually suggested that the $\mathrm{H}_{2} \mathrm{O}$ maser phase in $\mathrm{W} 49 \mathrm{~N}$ is associated with a strong stellar wind at $2000 \mathrm{~km} \mathrm{~s}^{-1}$.

The axial cavity revealed by the maser velocity curve in W49N may be our first direct glimpse of the collimation of a jet 
from a young stellar object. Even if the wind were collimated on a smaller scale, that information would be lost in a pressure-driven bubble, since the sound speed in the shocked wind gas is much higher than the expansion velocity. We must emphasize that this axial cavity provides the simplest explanation of the observations. Further observations and more selfconsistent theories may force more complex descriptions, possibly including the magnetic field effects that we neglected in our calculations. Icke et al. (1992) have recently proposed a similar confinement by a toroidal density distribution that is denser at the equator as an explanation for collimation of astrophysical jets. Masson \& Chernin (1992) come to a similar conclusion. Although they dismiss such confinement at distances of $\sim 1 \mathrm{pc}$ from the star, they consider such channeling possible closer to the star.

The observed ultracompact $\mathrm{H}$ II region in $\mathrm{W} 49 \mathrm{~N}$ must be trapped either in the wind or in the inner edge of the dense shell of shocked ambient gas in order for masing molecules to exist at the outer shock front. Recombinations in the wind trap $3 \times 10^{52} \dot{M}_{-3}^{2} v_{w, 8}^{-2} r_{11}^{-1}$ ionizing photons per second, where $r=$ $r_{11} \times 10^{11} \mathrm{~cm}$ is the wind's inner radius. Since this rate exceeds the likely emission rate of ionizing photons from the central star, the ionizing radiation is probably trapped by the wind. Recombination line measurements will not show large velocity widths because the ionized region is so compact. Measurement of the spectral shape of radio continuum emission, as done by Scoville et al. (1986), may reveal the wind and test our proposal.

The hundred year time scale that we find is less than $\sim 10^{-4}$ of the lifetime of an $\mathrm{O}$ star. Since there are less than $10^{4} \mathrm{O}$ stars in the galaxy, we expect to see very few other objects in this stage of evolution. Even the other stars in W49 are unlikely to have formed at so close to the same time that they would also be in the same several hundred year stage of their life. This may explain the exceptional nature of $\mathrm{W} 49 \mathrm{~N}$, the brightest $\mathrm{H}_{2} \mathrm{O}$ maser source in the galaxy, with an $\mathrm{H}_{2} \mathrm{O}$ isotropic luminosity of $\sim 1 L_{\odot}$. Note that typical $\mathrm{H}_{2} \mathrm{O}$ luminosities of $07-09$ stars are only $\sim 10^{-4} L_{\odot}$ (Genzel \& Downes 1979). If our model is correct, the uniqueness of the $\mathrm{W} 49 \mathrm{~N} \mathrm{H}_{2} \mathrm{O}$ maser stems from the fact that we have caught a massive $\left(\gtrsim 60 M_{\odot}\right)$ star during a rather brief phase of its evolution.

Our proposal implies that $\mathrm{H}_{2} \mathrm{O}$ masers trace the surfaces of the shells swept up by powerful winds around newly formed, massive stars. An estimate of the exact stage at which $\mathrm{H}_{2} \mathrm{O}$ maser activity turns on in a given source must involve a detailed calculation of the shell evolution combined with a maser model. A forthcoming detailed study by Elitzur, Hollenbach, \& McKee (1992) of the parameter space in which $\mathrm{H}_{2} \mathrm{O}$ maser emission occurs may provide the maser model. Interferometric maps of weaker sources will provide a detailed picture of the density distributions and wind strengths that produce the extraordinary range of maser luminosities observed (sources with $\mathrm{H}_{2} \mathrm{O}$ isotropic luminosities of $\lesssim 10^{-7} L_{\odot}$ have been detected). It is already known that $\mathrm{H}_{2} \mathrm{O}$ maser luminosity correlates with mass and spectral type of the central star (Genzel \& Downes 1979). These detailed maps of maser fields should provide our best description yet of the gas dynamics very close to newly formed stars.

We would like to thank Carl Gwinn, Jack Welch, and Kevin Zahnle for useful discussions. M.-M. M. L was supported by the Center for Star Formation Studies with funding from the NASA Astrophysical Theory program. M. E. acknowledges the award of Senior Research Associateship by the NRC and the partial support of NSF grant AST 90-16810.
Castor, J., McCray, R., \& Weaver, R. 1975, ApJ, 200, L107

Cowie, L. L. \& McKee, C. F. 1977, ApJ, 211, 135

Elitzur, M., Hollenbach, D. J., \& McKee, C. F. 1989, ApJ, 346, 983 (EHM) 1992 , in preparation

Felli, M., Palagi, F., \& Tofani, G. 1992, A\&A, in press

Genzel, R. 1986 in Masers, Molecules, and Mass Outflows in Star-Forming

Regions, ed. A. D. Haschick (Westford, MA: Haystack Obs.), 233

Genzel, R., et al. 1978, A\&A, 66, 13

Genzel, R., \& Downes, D. 1977, A\&AS, 30, 145

. 1979, A\&A, 72, 234

Gwinn, C. R., Moran, J. M., \& Reid, M. J. 1992, ApJ, submitted (GMR)

Icke, V., Mellema, G., Ballick, B., Euldrink, F., \& Frank, A. 1992, Nature, 355, 524

Kompane'ets, A. S. 1960, Soviet Phys. Dokl., 5, 46

\section{REFERENCES}

Königl, A. 1982, ApJ, 261, 115

Lada, C. J. 1985, ARA\&A, 23, 267

Mac Low, M.-M., \& McCray, R. 1988, ApJ, 324, 776

Mac Low, M.-M., Van Buren, D., Wood, D. O. S., \& Churchwell, E. 1991, ApJ, 369,395

Masson, C., \& Chernin, L. 1992, ApJ, 387, L47

Scoville, N., et al. 1986, ApJ, 303, 416

Steigman, G., Strittmatter, P. A. \& Williams, R. E. 1975, ApJ, 198, 575

Walker, R.C.,., et al. 1978, ApJ, 226, 95

Weaver, R., McCray, R., Castor, J., Shapiro, P., \& Moore, R. 1977, ApJ, 218, 377

Welch, W. J., Dreher, J. W., Jackson, J. M., Terebey, S., \& Vogel, S. N. 1987, Science, 238, 1550 